\title{
Research Paper: Psychometric Properties of Dunn's Sensory Profile School Companion
}

\author{
Guita Movallali ${ }^{1}$, Abas Nesayan ${ }^{2},{ }^{*}$ Roghayeh Asadi Gandomani ${ }^{2}$
}

1- Pediatric Neurorehabilitation Research Center, University of Social Welfare and Rehabilitation Sciences, Tehran, Iran. 2- Department of Psychology, Faculty of Humanities, University of Bojnord, Bojnord, Iran.

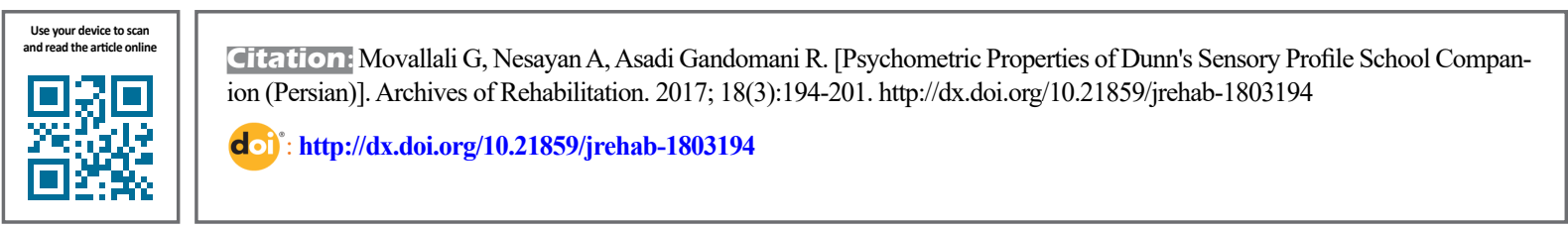

Received: 27 Apr. 2017 Accepted: 25 Jul. 2017

Keywords: Sensory profile, Validity, Reliability, School companion

\section{ABSTRACT}

Objective Sensory processing refers to reception, adjustment, and integration of sensory information sequentially and generates adaptive responses. People need to have appropriate sensory processing abilities for functioning adequately in the environment and be able to participate in activities of daily living. Dunn's sensory processing model consists of two constructions. The first construction involves neurological thresholds, and the second construction concerns self-regulation. When the two continuums are connected, four basic patterns of sensory processing appear. The four models are: sensation seeking (including representation of high thresholds and active self-regulation strategy), sensory avoiding (including representation of low thresholds and active self-regulation strategy), sensory sensitivity (including representation of low thresholds and passive self-regulation strategy), and low registration (including representation of high thresholds and passive self-regulation strategy). Sensory processing patterns can be seen in each age group from infancy to adulthood. These patterns are different for people with disabilities such as autism, attention deficit disorder/ hyperactivity disorder, schizophrenia, Asperger syndrome, developmental disorders, and learning disabilities compared to those without disability. Dunn sensory profile school companion is provided to assess sensory processing patterns in children. As sensory processing plays an important role in the daily life of people, the purpose of this study was to determine the validity and reliability of Dunn's sensory profile school companion in children and students in the age group of 3-11 years.

Materials \& Methods The study was a descriptive-analytical one. The population consisted of all children and students aged between 3 and 11 years and studying in Tehran schools in the academic year 94-95 and those who attended preschools. The sample consisted of 384 children and students who were selected through multi-stage cluster sampling. Dunn's sensory profile companion was used to collect data. Dunn's sensory profile companion is a standardized measurement tool for assessing sensory processing abilities of children and students in the classroom and school and preschool environment. This tool was built by Winne Dunn. This profile are using for age range from 3 years to 11 years and 11 months and be completed by teachers and educators. Descriptive statistics such as mean and standard deviation were used for data analysis and confirmatory factor analysis, and Cronbach's alpha was used to draw inferences from the data. Results Confirmatory factor analysis was used to assess the validity of the sensory processing profile school companion. The results showed that sensory processing profiles are school companion and four factors had construct validity $(\mathrm{P}<0.001)$. Internal consistency (Cronbach's alpha) was used to evaluate the reliability. Cronbach's alpha for the entire questionnaire was 0.879 , and for sensory registration, sensation seeking sensory sensitivity and sensory avoiding was $0.818,0.885,0.825,0.812$ respectively. Thus, this confirms that the sensory processing profile school companion and four factors are reliable. Conclusion The results showed that Dunn's sensory profile has good reliability and validity. Dunn's sensory profile is a useful tool for assessing sensory processing patterns in school and kindergarten settings, and can be used by occupational therapists in clinical environments and by psychologists in educational environments. Information obtained from this profile can have diagnostic value and could also be used for the design of curriculum and classroom space.

\section{* Corresponding Author:}

Roghayeh Asadi Gandomani, PhD

Address: Department of Psychology, Faculty of Humanities, University of Bojnord, Bojnord, Iran.

Tel: +98 (58) 32201000

E-Mail: r.asadi@ub.ac.ir 


\title{
بررسى ويرٔكى هاى روانسنجى نيمرخ حسى دان فرم مدرسه
}

\author{
كيتا موللى'، عباس نسائيان؛ •رقيه اسدى كندمانى'
}

ا - مركز تحقيقات توانبخشى اعصاب اطفال، دانشُكاه علوم بهزيستى و توانبخشى، تهران، ايران.

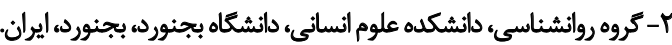

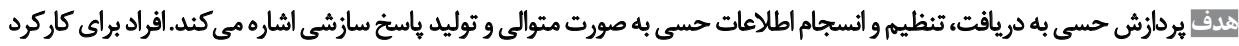

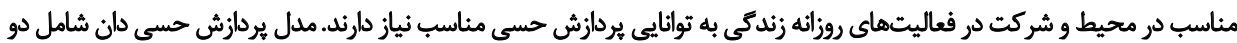

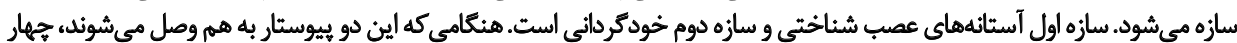

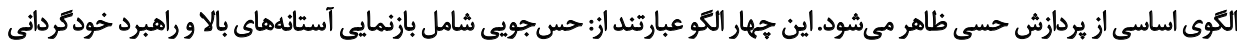

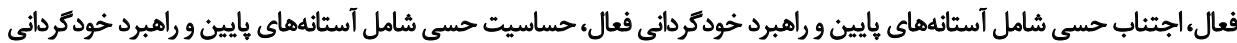

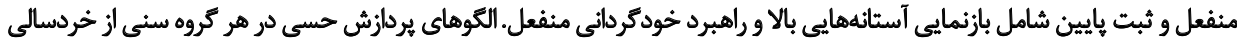

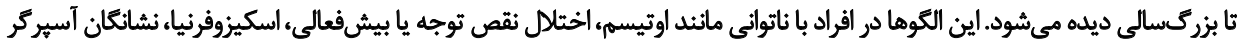

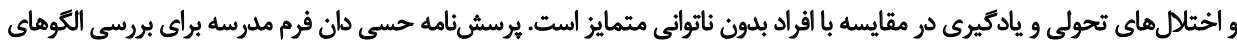

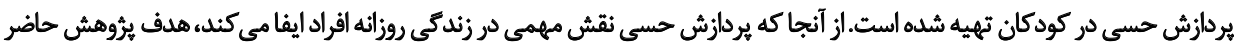

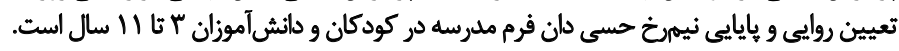

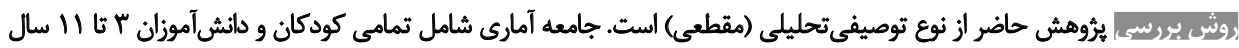

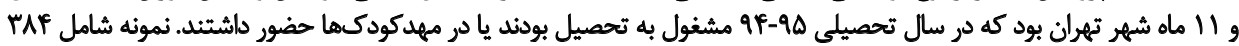

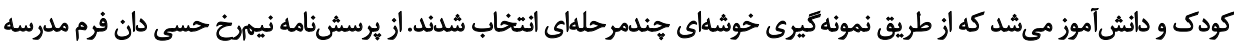

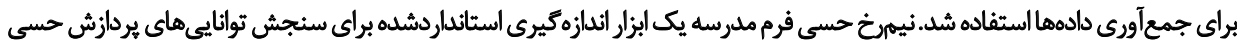

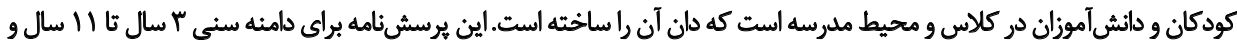

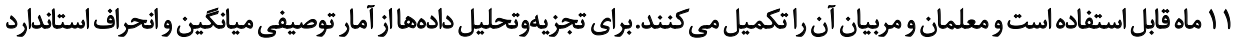

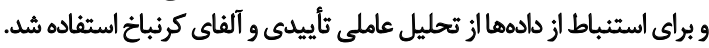

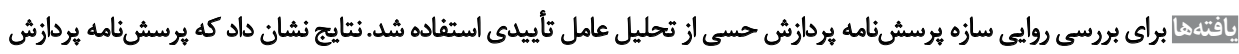

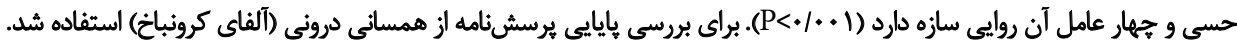

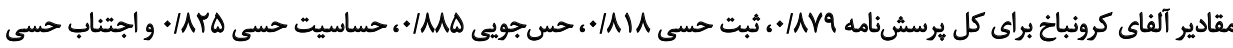

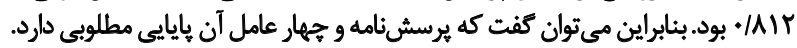

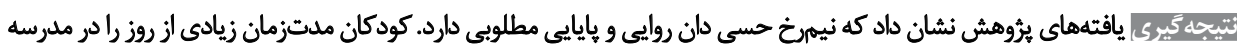

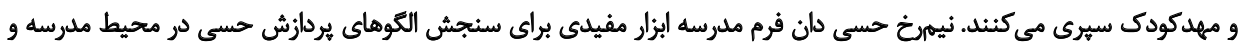

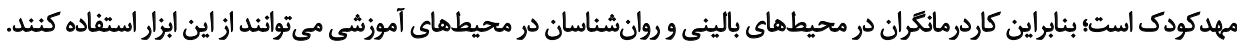

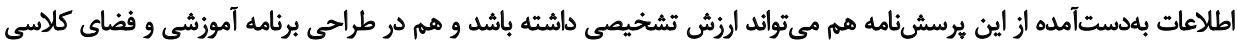

اجازه ميدهد آنجه را كه لذتبخش است، جستوجوكنيم و از

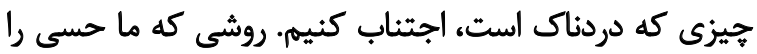

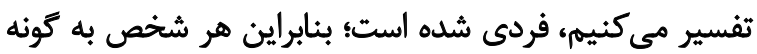

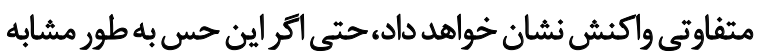

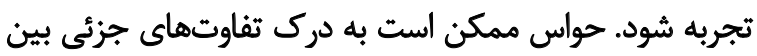

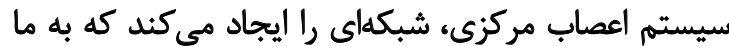

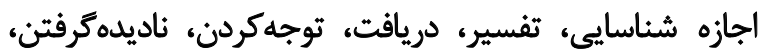

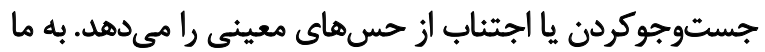


اجرايى و الكوهاى يردازش حسى در كودكان اوتيسم يرداختند.

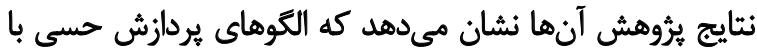

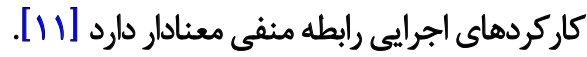

مدل بردازش حسى دان شامل دو سازه است. سازه اول

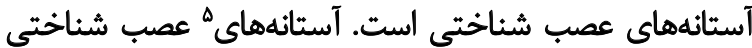

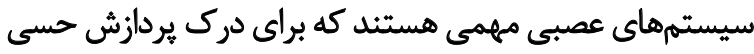

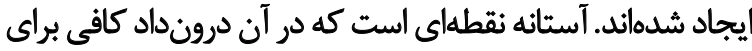

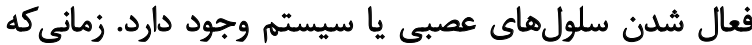

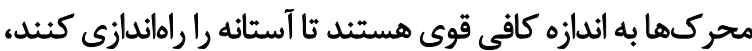

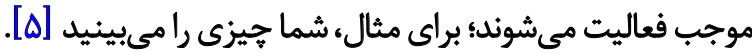

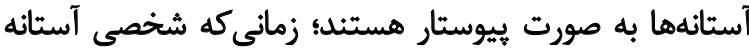

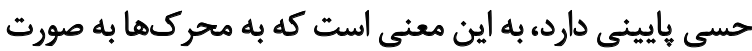

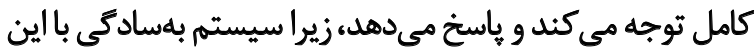

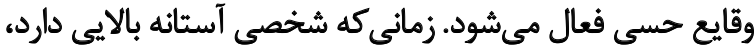

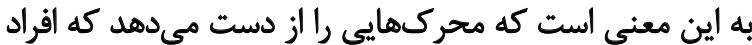

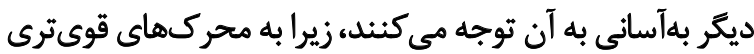

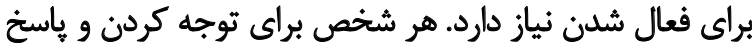

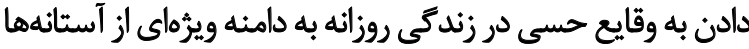

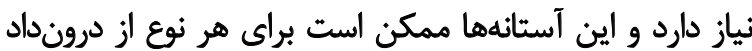

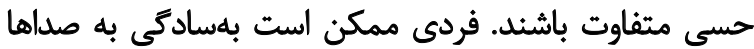

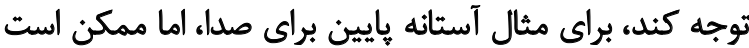

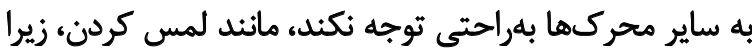

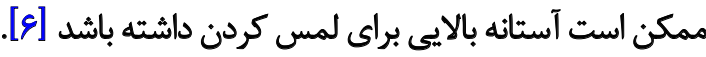

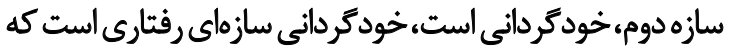

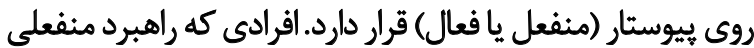

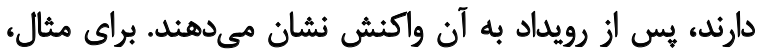

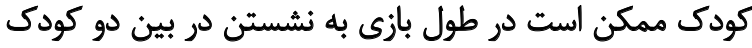

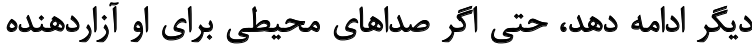

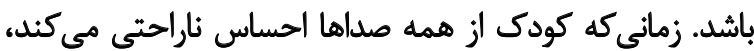

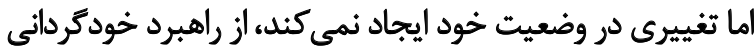

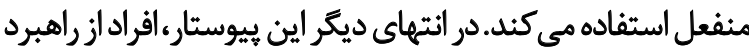

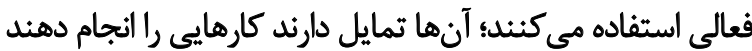

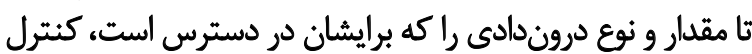

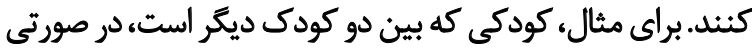

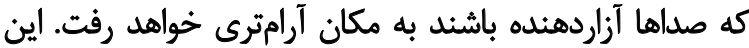

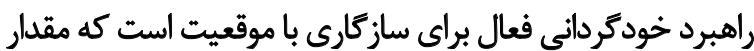

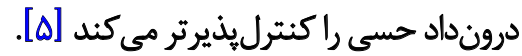

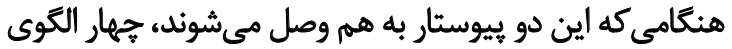

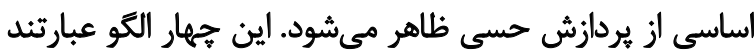

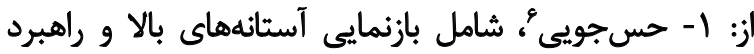

5. Threshold

6. Sensation seeking

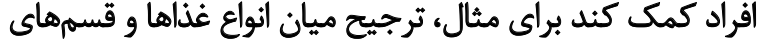

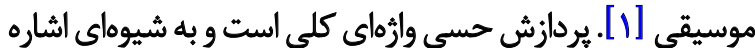

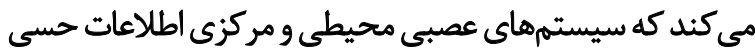

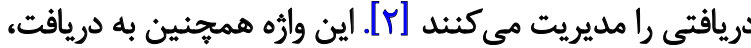

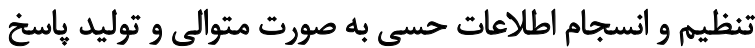
سازشى اشاره مى كند [ب]. تلاش محققان براى توسعه مطالعه

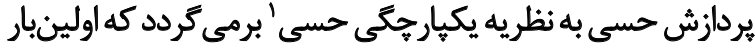

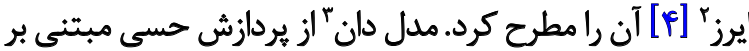

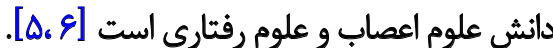

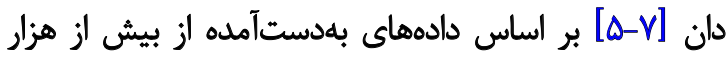

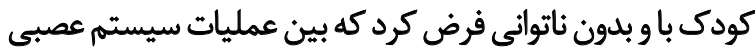

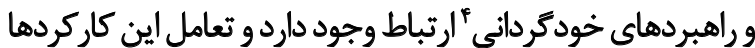

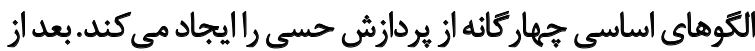

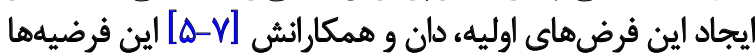

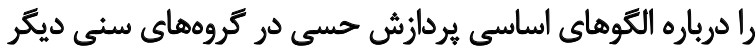

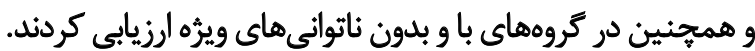

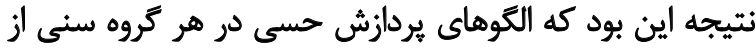

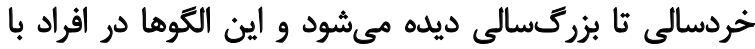

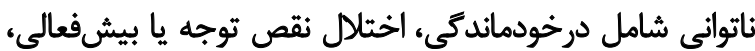

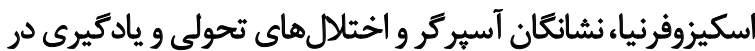

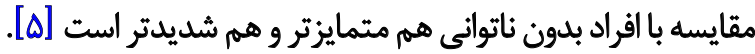

در ايران نيز يُووهشهايى در زمينه بردازش حسى بر اساس

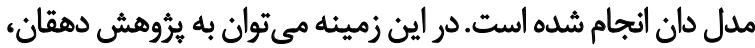

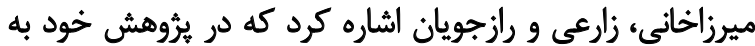

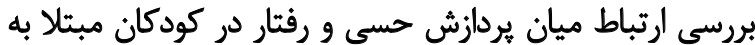

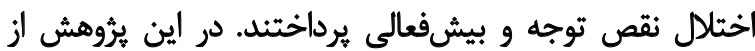

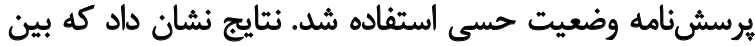

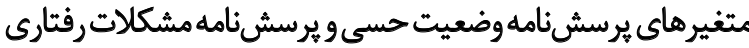

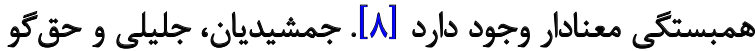

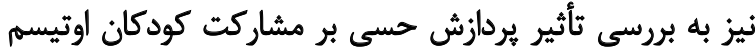

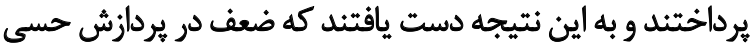

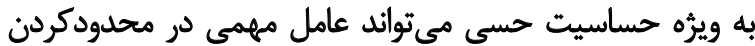

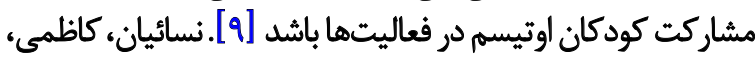

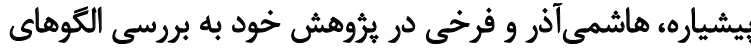

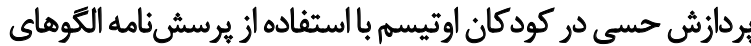

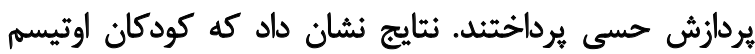

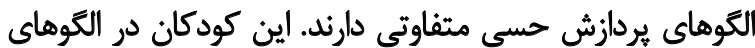

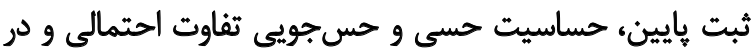

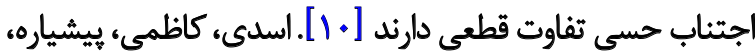

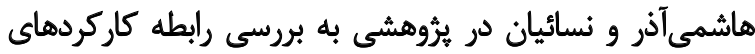

1. Sensory integration theory

2. Ayres

3. Dunn's Model

4. Self-regulation 
جدول ا. مدل يردازش حسى دان [ه]]

راهبرد خودكردانى /ياسخ هاى رفتارى

\begin{tabular}{|c|c|c|}
\hline آستانههاى عصب شناختى & منفعل & 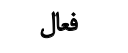 \\
\hline آستانه بالا & ثبت بايين & حسجويى \\
\hline آستانه هايين & حساسيث حسى & اجثناب حسيى \\
\hline
\end{tabular}

توانبخننى

از روش نمونهكيرى خوشهاى جند مرحلداي، نمونه انتخاب شد.

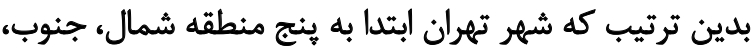

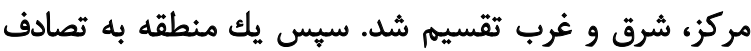

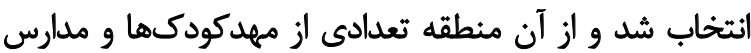
بررسى شدند تانمونه مدنظر حاصل شود.

ابزوار

دان (ع +. (T)، نيمرخ حسى فرم مدرسه را تهيه كرده است.

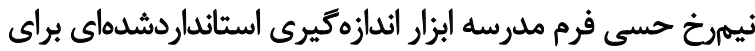

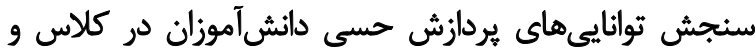

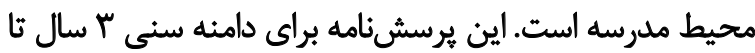
II سال و II ماه قابل استفاده است [1F].

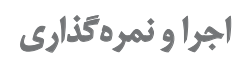

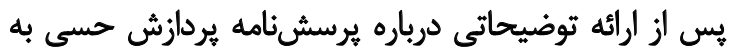

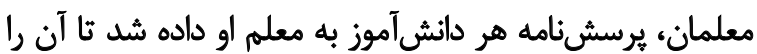

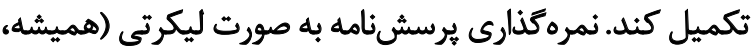

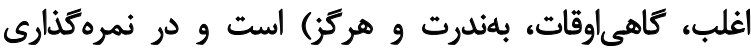

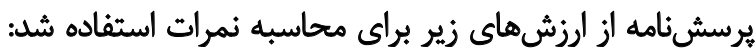

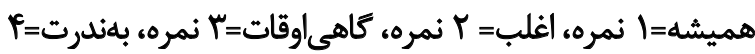

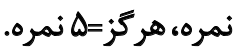

احر معلم بين دو طبقه راعلامت زد، نمره طبقه بالاتر گزارش

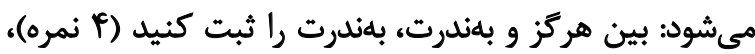

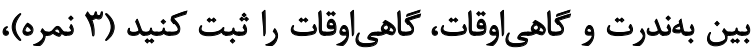

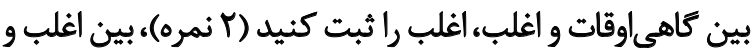
هميشه، هميشه راثبت كنيد (ا نمره).

$$
\text { وأش الجرا }
$$

يس از اخذ مجوزهاي لازم، نمونه از بين جامعه هدف انتخاب

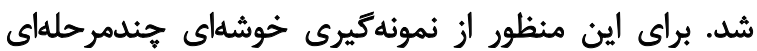

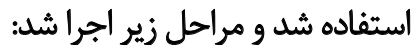

با توجه به ماهيت كار، ئروهش حاضر در دو مرحله انجام شد.

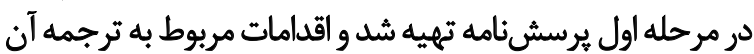

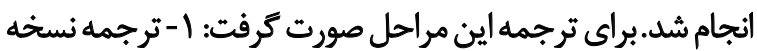

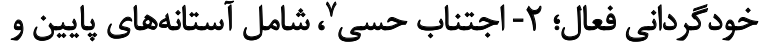

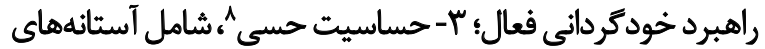

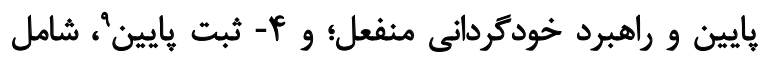

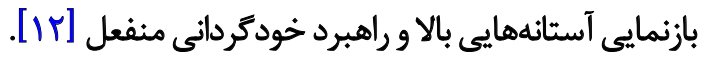
دان براى سنجش نيهرخ حسى يرسشنامههاي بسيارى تهيه

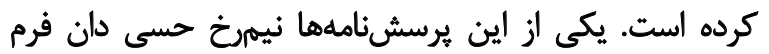

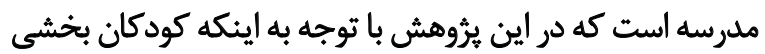

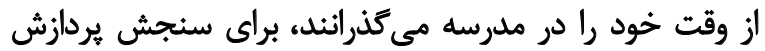

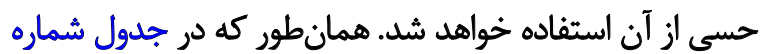

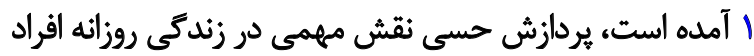

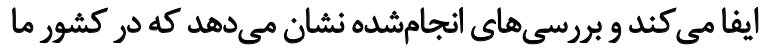

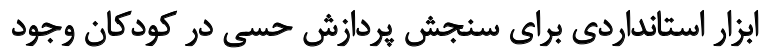

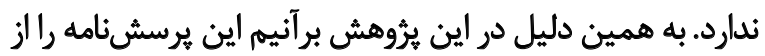

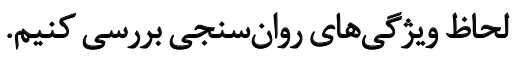

$$
\text { ووش بروسي }
$$

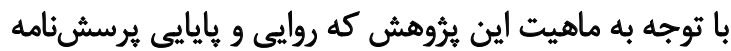

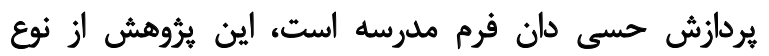

$$
\text { توصيفى تحليلى (مقطعى) محسوب ملى مدرده است اين }
$$

$$
\text { جامعله، نمونه و روش نمونهيّيرى }
$$

جامعه اين يثوهش را تمامى كودكان ب تا Iل ال سال شهر تهران

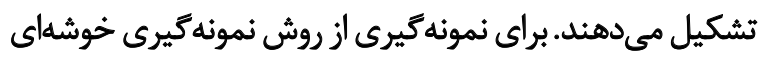

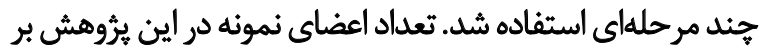

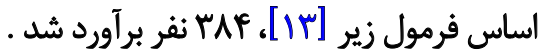

$$
n=\frac{z^{2}{ }^{2} p q}{d^{2}}=384
$$

با توجه به اينكه محقق از مقدار حقيقى Pاطلاع ندارد، مي تواند

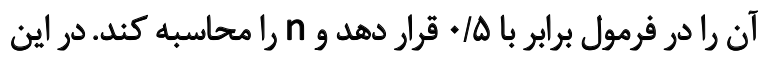

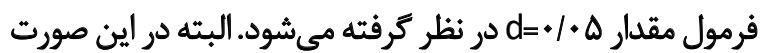

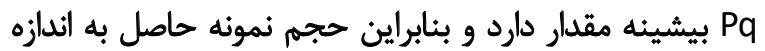

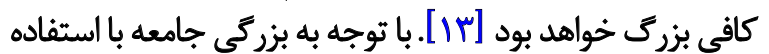




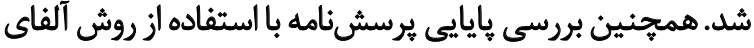

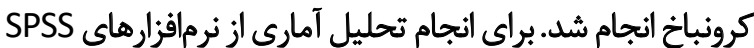

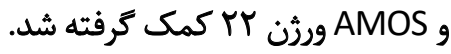

يافتهان

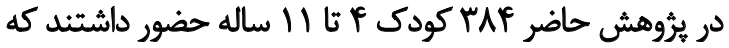
| iV|

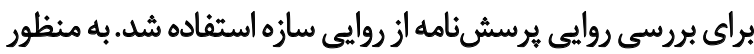

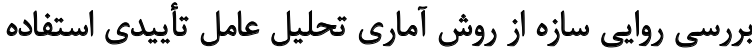

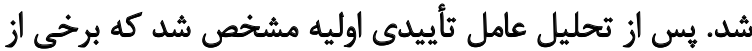

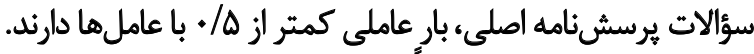

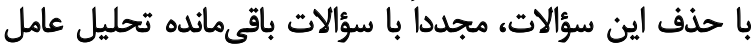

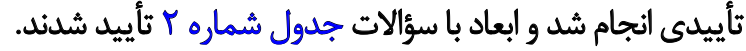
با توجه به جدول شماره "ا، در همه ابعاد، نمره كل يرسش نامهاه، بهزج

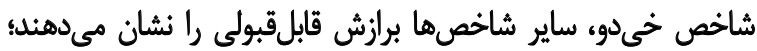

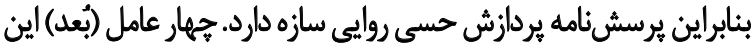

انكليسى به فارسي Y- تهيه نسخه واحد از نسخههاى ترجمهشده

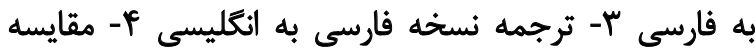

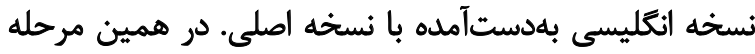

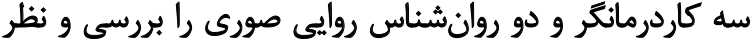

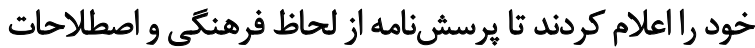

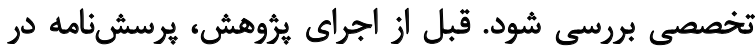

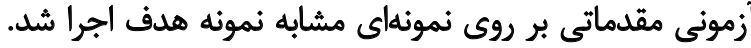

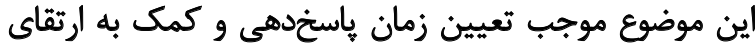

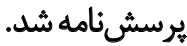

در مرحله دوم يرسشنامه تهيهشده روى نمونه مدنظر اجرا

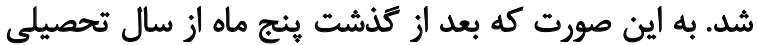

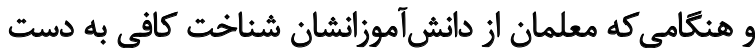

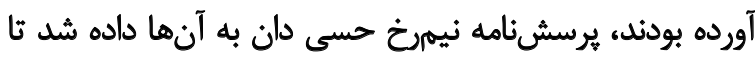

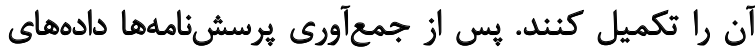

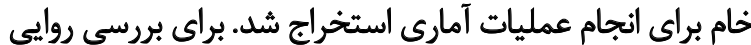

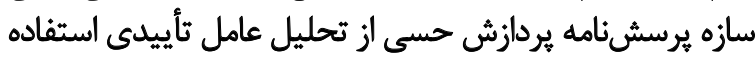

جدول r. سؤلات و بار عاملى آنها با ابعاد هرسشنامه

\begin{tabular}{|c|c|c|c|c|}
\hline بار عاملى & سؤال & بار عاملى & سؤال & عامل \\
\hline $1 / 91$ & ז" &.$/ Y A Y$ & 1 & \multirow{7}{*}{ ثبت حسى } \\
\hline . $/ \Delta \Delta A$ & 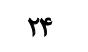 &.$/ N 1$ & $r$ & \\
\hline.$/ 991$ & TD & . Mas & $r$ & \\
\hline.$/ 8$ & rg & . $/ 41$ & 11 & \\
\hline . /qar & is & . rvar & ir & \\
\hline . & 19 & . /VDP & ir & \\
\hline . & $\Delta$. & I/gW & $\pi$ & \\
\hline$. / \Delta+1$ & $r$. &.$/ 8.4$ & $r$ & \multirow{4}{*}{ حسجويى } \\
\hline זוv/. & $r q$ &.$/ P A 1$ & rq & \\
\hline t/999 & $\varphi$. & $\cdot / \lambda \cdot 1$ & $r v$ & \\
\hline$\cdot / N \cdot \Delta$ & il & $\cdot / v a$ & $M$ & \\
\hline.$/(29)$ & 9 & $\cdot / A_{-1}$ & $\Delta$ & \multirow{4}{*}{ ساسيت حسى } \\
\hline$. / \Delta \Delta \mid$ & 19 & . /Far & 9 & \\
\hline$\cdot / \Delta \cdot v$ & 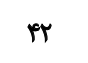 &.$/ 1998$ & $v$ & \\
\hline- & - & . /pye & $\wedge$ & \\
\hline.$/ \Delta .1$ & $\Delta \mathrm{A}$ &.$/ 819$ & r & \multirow{6}{*}{ اجتاب حسى } \\
\hline s"ه & $\Delta 9$ & . Ars & $\pi$ & \\
\hline ./ATY & \&. & . /rar & $\pi$ & \\
\hline . NGT & 91 & - /agr & $\pi$ & \\
\hline . & gr & . & ro & \\
\hline- & - &.$/ \% \Delta \varphi$ & $\Delta V$ & \\
\hline
\end{tabular}

توانبخننى 
جدول "ا. بررسى شاخصهاي برازش تحليل عامل تأييدى برسشئامه بردازش حسى (ابعاد و نمره كل)

\begin{tabular}{|c|c|c|c|c|c|}
\hline نتيجه & دامنه قابلقبول & مقدار احتمال & آماره & شاخص & ابعاد \\
\hline برازش مناسب ثيست & مقدار الحتمال بيشتر از ه+/. & $<+/+1$ & $r q_{+} / 11$ & $X^{r}$ & \multirow{4}{*}{ 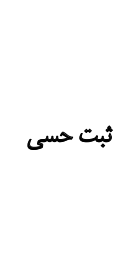 } \\
\hline برازش مناسب است & بالاتم از 9/. & - &.$/ 91$ & CFI & \\
\hline برازش مناسب است & بالاتم از 9/. & &.$/ 91$ & GFI & \\
\hline برازش مناسب است & كمتر أز 1٪/. & - & $* * n$ & RMSEA & \\
\hline برازش مناسب نيست & مقدار الحتمال بيشتر از هـ/. & $<* / \bullet 1$ & 181 & $X^{r}$ & \multirow{4}{*}{ حساسيت حسى } \\
\hline برازش مناسب است & بالاتم از 9/. & - &.$/ 9 \%$ & CFI & \\
\hline برازش مثاسب است & بالاتر از 9/. & &.$/ 9$ & GFI & \\
\hline برازش مناسب است & كمتر از ب+|. & - & .1 .98 & RMSEA & \\
\hline برازش هناسب نيست & هقدار الحتمال ييشتر از هـ/. & $<. / . .1$ & 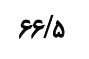 & $X^{r}$ & \multirow{4}{*}{ حسجويى } \\
\hline برازٔش مناسب است & بالاتر از ج/. & - &.$/ 91$ & CFI & \\
\hline برازش مناسب است & بالاتر از ج/. & &.$/ 9$ & GFI & \\
\hline برازش مناسب است & كمتر الز A•/. & - & $\%$. vo & RMSEA & \\
\hline برازٔش مناسب نيست & مقدار احتمال بيشتر از هـ/. & $<* / \bullet 1$ & 181 & $X^{r}$ & \multirow{4}{*}{ اجتناب حسى } \\
\hline برازش مناسب است & بالاتر از 9/. & - &.$/ 9 \%$ & CFI & \\
\hline برازش مناسب است & بالاتم از 9/. & &.$/ 9$ & GFI & \\
\hline برازش مناسب است & كمتر الز +1+| & - & .1 .98 & RMSEA & \\
\hline برازش مناسب نيست & مقدار احتمال بيشتر از هـ/. & $<+/+1$ & rED/Tr & $X^{r}$ & \multirow{4}{*}{ ن ممرهكل } \\
\hline برازش مناسب است & بالاتتر از 9/. & - &.$/ 9 r$ & CFI & \\
\hline مبرازش مناسب است & بالاتر از ج/. & &.$/ 9 \%$ & GFI & \\
\hline برازش مناسب است & كمتر الز م+|. & - & .1 .90 & RMSEA & \\
\hline
\end{tabular}

جايايى يرسش نامه تأييدشده، از همسانى درونى (آلفاى كرونباخ) استفاده

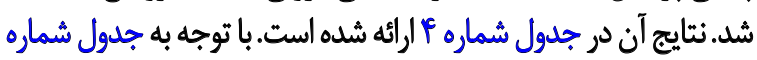

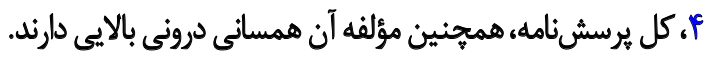

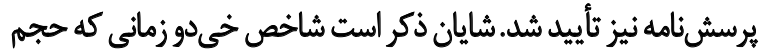

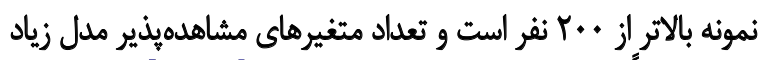

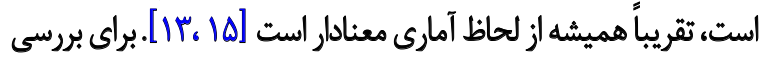

جدول ع. بروسي بايايي ثبرسشنامه با استفاده از روش همسانى دروني (آلفاى كرونباخ)

\begin{tabular}{|c|c|c|}
\hline ميزان آلفا & تعداد كويه & \\
\hline ./AYq & po & كل يرسش نامه \\
\hline.$/ A M$ & if & عامل (ثبت حسى) \\
\hline . MAs & $\wedge$ & عامل ب (حس جويیى) \\
\hline - /AYA & r & عامل ب (حساسيت حسى) \\
\hline ./AIr & $m$ & عامل f(اجتناب حسى) \\
\hline
\end{tabular}


نتايج نيمرخ حسى فرم مدرسه مي تواند با يافتههاى بهدست آمده

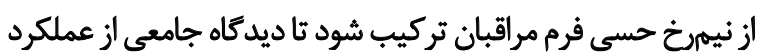

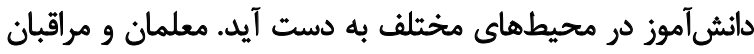

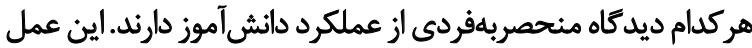

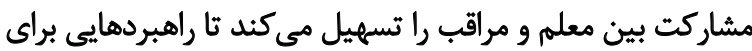

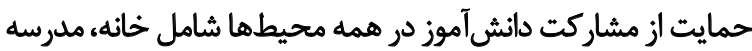

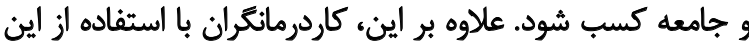

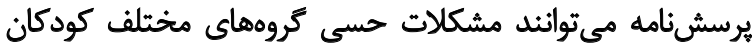

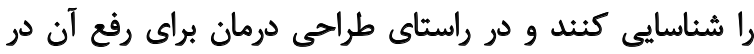
محيطهاى بالينى برنامهريزى و اقدام كنيند.

\section{نتيجليَيرى}

از مقياس مذكور مى توان براي اين موارد استفاده كرد: () سنجش

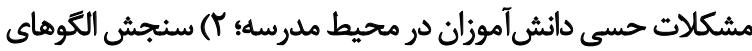

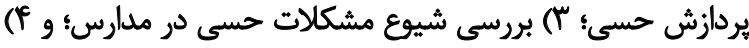

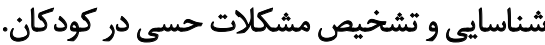

يكى از محدوديتهاي اين يُروهش همكارىنكردن برخى از

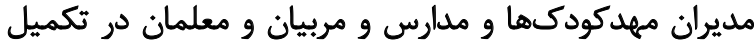

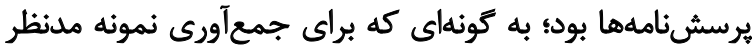

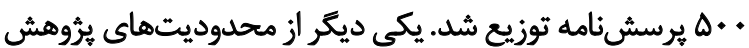

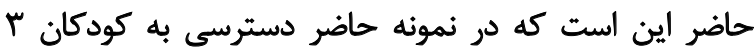

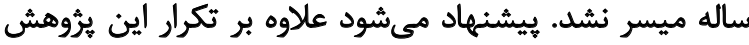

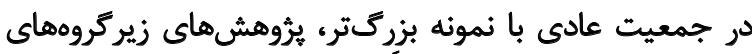

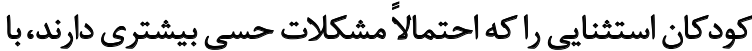

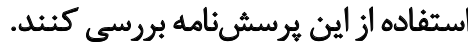

$$
\text { تشكر و قدرداثي }
$$

از تمامى معلمان و مربيائى كه ما را در تكميل يرسشنامهاهها يارى

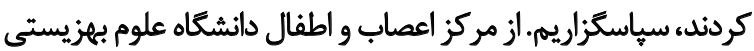

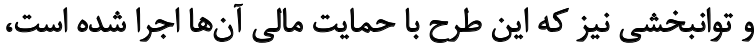

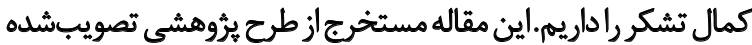
در مركز تحقيقات اعصاب و اطفال دانشكاه علوم بهزيستى است.
ث

در دهلهاى اخير براى تهيه ابزارهاى معتبر در زمينه اندازهكيرى

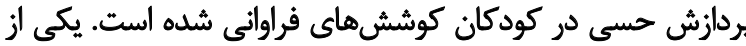

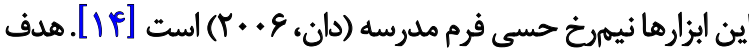

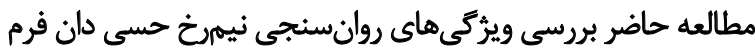

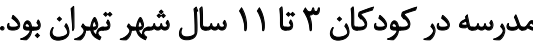

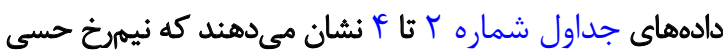

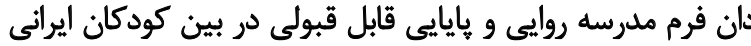

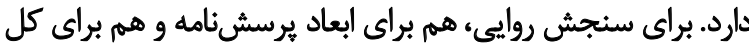

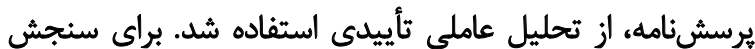

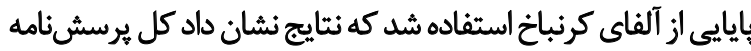

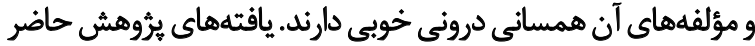

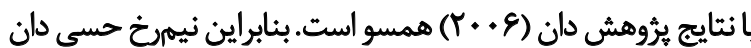

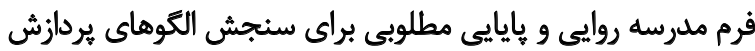
حسى كودكان در محيط مدرسه دارد.

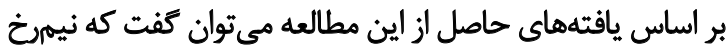

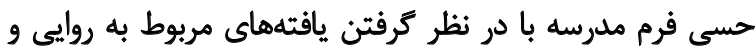

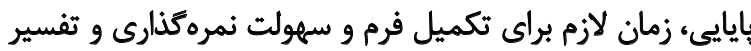

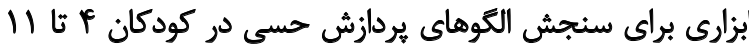
سال كاملاً مناسب است.

يكيارجّكى حسى عبارت است از توانايى مغز در دريافت، تشخيص،

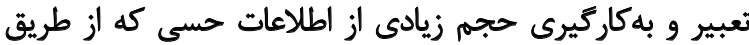

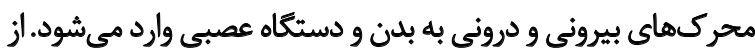

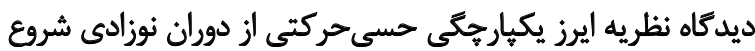

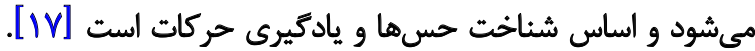

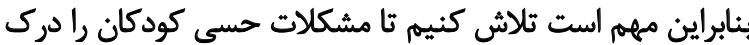

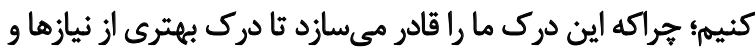

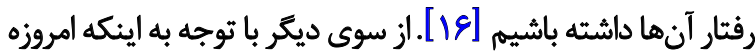

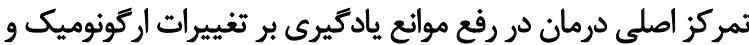

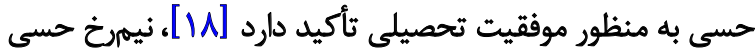

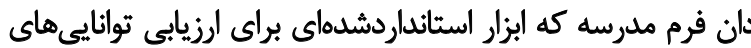

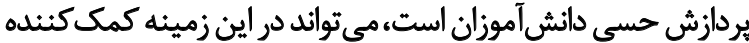

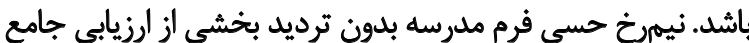

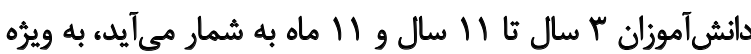

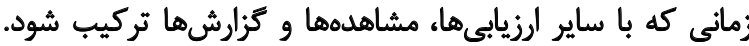

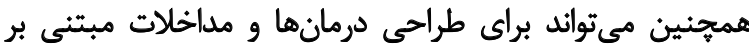

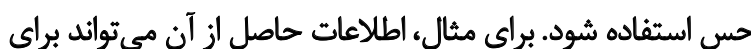

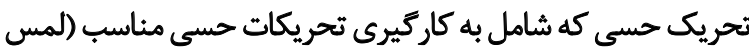

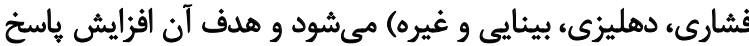

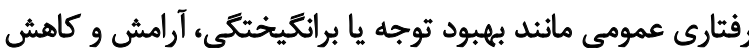

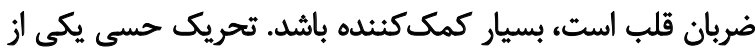

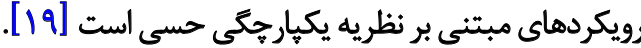




\section{References}

[1] Myles BS. Asperger syndrome and sensory issues: Practical solutions for making sense of the world. Lenexa: Autism Asperger Publishing Company; 2000.

[2] Miller LJ. Sensational kids: Hope and help for children with Sensory Processing Disorders (SPD). Canada: Perigee Book; 2007.

[3] Schaaf RC, Miller LJ. Occupational therapy using a sensory integrative approach for children with developmental disabilities. Mental Retardation and Developmental Disabilities Research Reviews. 2005; 11(2):143-8. doi: 10.1002/mrdd.20067

[4] Brown NB, Dunn W. Relationship between context and sensory processing in children with autism. American Journal of Occupational Therapy. 2010; 64(3):474-83. doi: 10.5014/ajot.2010.09077

[5] Dunn W. Supporting children to participate successfully in everyday life by using sensory processing knowledge. Infants \& Young Children. 2007; 20(2):84-101. doi: 10.1097/01. iyc.0000264477.05076.5d

[6] Dunn W. The impact of sensory processing abilities on the daily lives of young children and their families: A conceptual model. Infants \& Young Children. 1997; 9(4):23-35. doi: 10.1097/00001163-199704000-00005

[7] Dunn W, Myles BS, Orr S. Sensory processing issues associated with asperger syndrome: A preliminary investigation. American Journal of Occupational Therapy. 2002; 56(1):97-102. doi: 10.5014/ajot.56.1.97

[8] Dehghan F, Mirzakhani N, Alizade Zare M, Razjoyan K. [The relationship between sensory processing and behavior in children with attention deficit disorder and hyperactivity 7 to 10 years old (Persian)]. Journal of Modern Rehabiliation. 2015; 9(3):9-18.

[9] Jamshidian E, Jalili N, Haghgoo H. [The effect of sensory processing abilities on participation of children with autism (Persian)]. Medical Daneshvar. 2016; 23(120):33-44.

[10] Nesayan A, Kazemi F, Pishyare E, Hashemi Azar J, Farokhi NA. [Sensory processing patterns of Autistic children from teachers' point of view (Persian)]. Journal of North Khorasan University of Medical Sciences. 2013; 5(3):653-61.

[11] Asadi Gandomani R, Kazemi F, Pishyare E, Hashemi Azar J, Nesayan A. [Relationship between executive functions with sensory processing patterns in autistic student (Persian)]. Psychology of Exceptional Individuals. 2016; 6(23):27-48. doi: 10.22054/ jpe.2016.5282

[12] Hochhauser M, Engel-Yeger B. Sensory processing abilities and their relation to participation in leisure activities among children with High-Functioning Autism Spectrum Disorder (HFASD). Research in Autism Spectrum Disorders. 2010; 4(4):746-54. doi: 10.1016/j.rasd.2010.01.015

[13] Hooman H. [Structural equation modeling using LISREL software (Persian)]. Tehran: SAMT Publication; 2005.

[14] Dunn W. Sensory Profile School Companion (SPSC). London: Pearson; 2006.

[15] Abareshi A, Hosseini Y. [Structural equation modeling (Persian)]. Tehran: Jame'e Shenasan; 2011.
[16] Kern JK, Trivedi MH, Garver CR, Grannemann BD, Andrews AA, Savla JS, et al. The pattern of sensory processing abnormalities in autism. Autism. 2006; 10(5):480-94. doi: $10.1177 / 1362361306066564$

[17] Ghasem-Pour L, Hosseini FS, Mohammad-Zadeh H. [Does sensory-motor integration exercises effect on static and dynamic balance in children with trainable mental retardation (Persian)]. Archives of Rehabilitation. 2015; 16(1):26-35.

[18] Matin Sadr N, Haghgoo HA, Samadi SA, Rassafiani M, Bakhshi E. [Research paper: Impact of air seat cushions and ball chairs on classroom behavior of students with autism spectrum disorder (Persian)]. Archives of Rehabilitation. 2016; 17(2):136-47. doi: 10.21859/jrehab-1702136

[19] Gharebaghi S, Mohhamad Jani Z, Sortchi H, Biglariyan A. [Effectiveness of sensory stimulation by weighted vest on motor skills and attention of 6-10 years old children with learning disorders (Persian)]. Archives of Rehabiliation. 2011; 12(2):27-31. 